\title{
Das Psychiatriejahr in der Ausbildung zum Psychologischen Psychotherapeuten
}

\begin{abstract}
Das Inkrafttreten des Psychotherapeutengesetzes (PsychThG) liegt nun 10 Jahre zurück, und das "Forschungsgutachten zur Ausbildung zum Psychologischen Psychotherapeuten und zum Kinder- und Jugendlichenpsychotherapeuten» wurde kürzlich vorgelegt. Die damit verbundenen aktuellen Diskussionen um die Ausbildung zum Psychologischen Psychotherapeuten nimmt Verhaltenstherapie zum Anlass, in dem neuen Format der Pro-und-ContraDebatte das in der Ausbildung verankerte Psychiatriejahr einer kritischen Würdigung zu unterziehen. Dazu werden die beiden Herausgeber Prof. Fritz Hohagen (Lübeck) und Prof. Winfried Rief (Marburg) als Vertreter ihrer jeweiligen Disziplinen (Psychiatrie und Psychotherapie bzw. Klinische Psychologie) und Berufsgruppen (Diplompsychologen, Psychologische Psychotherapeuten bzw. Fachärzte für Psychiatrie und Psychotherapie) ihren Standpunkt erläutern.
\end{abstract}

Fritz Hohagen, Lübeck

\section{Contra}

Die Monopolstellung der Psychiatrie für die Psychotherapieausbildung ist nicht gerechtfertigt

Kann man wirklich gegen das Psychiatriejahr im Rahmen der Ausbildung von Psychotherapeuten sein? Ich war selbst mehrere Jahre in der Psychiatrie tätig und erinnere mich durchaus gerne an wichtige Berufserfahrungen, die meinen weiteren Werdegang geprägt haben. Deshalb darf der nachfolgende Beitrag auf keinen Fall fehlverstanden werden als ein Plädoyer gegen die Praxiserfahrungen, die man in der Psychiatrie sammeln kann. Er muss jedoch eindeutig so verstanden werden, dass die Monopolstellung, die das Psychotherapeutengesetz der Praxiszeit in der Psychiatrie zubilligt, in dieser Form nicht nur überzogen und ungerechtfertigt ist, sondern auch zu Missbrauch verleitet hat.

Im Psychotherapeutengesetz (PsychThG) wird festgelegt, dass zur Psychotherapieausbildung mindestens $1 \mathrm{Jahr}$ (1200 h) in einer psychiatrischen Einrichtung Erfahrungen gesammelt werden müssen. Damit ist dieses «Psychiatriejahr» der größte Einzelposten im Katalog der geforderten Leistungen für die Psychotherapieausbildung. Im Vergleich zu den vielen anderen Einrichtungen, die Psychotherapie anbieten und in denen Psychotherapeuten lernen könnten, wird die Psychiatrie herausgestellt und es wird ihr eine Monopolstellung eingeräumt.

Monopolstellungen werden fast immer missbraucht. So ist es auch mit dem Psychiatriejahr geschehen: Mit fadenscheinigen Argumenten wurde behauptet, dass die Teilnehmer keine Entlohnung erhalten könnten, und die Ausbildungsteilnehmer (PPiA) hatten zumindest in einigen Bundesländern keine andere Wahl, als darauf einzugehen. Da zudem die inhaltliche Gestaltung des Psychiatriejahres nur wenig festgelegt war, wurden viele Diplompsychologinnen und Diplompsychologen als billige Arbeitskräfte missbraucht, die volle Dienstleistung erbringen mussten und bei den Statistiken für die Psychiatrie-Personalverordnung (PsychPV) als psychologisches Personal gewertet wurden. Manches gruppentherapeutische Programm in Psychiatrien, manche PsychiatrieAmbulanz funktioniert nur durch den Einsatz von PPiA. Ihre Vorbildung als Diplompsychologen sowie ihre Vorkenntnisse durch diverse Praktika im Psychologiestudium wurden also fachlich ausgenutzt, jedoch vertraglich nicht mit Entlohnung und sozialer Sicherstellung gewürdigt. Zwar gibt es selbstverständlich auch positive Beispiele, aber es bestätigt sich auch hier, dass Monopolstellungen zu Missbrauch führen.

Immer wieder hört man als Begründung für das Psychiatriejahr, dass angehende Psychotherapeuten dort mit Störungsbildern vertraut gemacht werden sollen, in denen sie keine Psychotherapie anwenden sollen. Dies mag einen

\section{KARGER}

Fax +497614520714

Information@Karger.de

www.karger.com (c) 2009 S. Karger GmbH, Freiburg

Accessible online at:

www.karger.com/ver 
gewissen Sinn ergeben, aber muss der größte Brocken der Psychotherapieausbildung damit verbracht werden zu lernen, was man nicht tun soll? Dies begründet definitiv keine 12-monatige Mitarbeit, sondern würde gegebenenfalls ein 2-monatiges Praktikum rechtfertigen. Ein solches hat die Mehrzahl der PPiA jedoch bereits im Studium absolviert.

Ein weiteres Argument für das Psychiatriejahr ist, dass in der Psychiatrie eine gewisse Vielfalt an Störungsbildern vorkommt und sie somit die besten Lernbedingungen bietet. Wie in einer Untersuchung der Arbeitsgruppe von Prof. Koch hingegen festgestellt wurde, überlappen sich die Diagnosestatistiken von Akutpsychiatrien und anderen stationären Psychotherapie-Einrichtungen zu $80 \%$ und zeigen sehr ähnliche Diagnosehäufigkeiten [Schulz et al., 2008]. Dabei sind in den Psychiatrien Patientengruppen unterrepräsentiert, die jedoch für die nachfolgende psychotherapeutische Praxis von höchster Relevanz sind (z.B. Patient/innen mit Essstörungen oder chronischen Schmerzsyndromen). Da zudem viele PPiA als Arbeitskräfte in den Psychiatrien eingesetzt werden, machen sie häufig nur Erfahrungen in 1-2 Spezialabteilungen. Von einem Kennenlernen der gesamten Bandbreite psychiatrischer Diagnosen kann somit oft keine Rede sein.

Schließlich bleibt noch das Argument, dass im stationären Setting Patienten behandelt werden, die wesentlich schwerer erkrankt sind als die meisten Patienten in einem ambulanten Setting. Dem würde ich zwar zustimmen, diese Aussage jedoch erweitern auf alle stationären Settings mit einem intensiven psychotherapeutischen Programm. Ich habe während meiner Zeit in einer psychosomatischen Fachklinik deutlich mehr Erfahrungen gesammelt mit Extremformen von Borderline-Persönlichkeitsstörungen, mit «house-bound Agoraphobics» und mit Anorexie-Patientinnen in lebensbedrohlichem Zustand als in meiner Zeit in der Psychiatrie. Schließlich sollte auch nicht vergessen werden, dass auch Patienten mit schwersten psychischen Störungen jeweils nur kurze Zeitspannen stationär behandelt werden und deutlich länger durch ambulante Versorgungsstrukturen getragen werden. Die meisten stationären Patienten sind vor und nach dem stationären Aufenthalt ambulante Patienten, und angehende Psychotherapeuten müssen lernen, auch schwergestörte Patienten im ambulanten Setting unterstützen zu können.

Schließlich könnte man noch als Argument anbringen, dass es ja um die Psychotherapieausbildung geht und behaupten, man könne Psychotherapie am besten in Psychiatrien lernen. Es ist gut, dass es zwischenzeitlich wirklich Psychiatrische Kliniken gibt, die auch ein anerkennenswertes psychotherapeutisches Programm anbieten. Dem stehen jedoch zahlreiche psychiatrische Einrichtungen gegenüber, bei denen entweder keine strukturelle Verankerung von Psychotherapie zu erkennen ist oder nur auf einem Stand, den man nicht mit moderner Psychotherapie in Verbindung bringen kann. Grundvoraussetzungen für eine gute Struktur- und Prozessqualität psychotherapeutischen Handelns liegen nicht in allen Psychiatrien vor. In manchen Psychiatrien werden vollwertig ausgebildete
Diplompsychologen mit guten psychotherapeutischen Grundkenntnissen Stationsärzten unterstellt, deren psychotherapeutische Kompetenz hier nicht näher diskutiert werden soll, statt dass die Klinik psychotherapieerfahrene Supervisoren vorhalten würde. An anderen Orten werden Veranstaltungen als «Supervision» bescheinigt, die qualitativ weit entfernt sind von den erwünschten Supervisionsgruppen mit maximal 4 Teilnehmern und einem Psychotherapie-erfahrenen Anleiter. Trotz mancher positiver Veränderungen in diesem Bereich in den letzten Jahren hat sich die Psychiatrie historisch mit der Psychotherapie sehr schwer getan, so dass in anderen stationären Einrichtungen (z.B. Psychosomatische Kliniken, Suchtkliniken) Psychotherapie schon länger verankert ist und zum Teil auf qualitativ höherwertigem Niveau liegt.

Die Regelungen des PsychThG zum Psychiatriejahr haben somit dazu geführt, dass eine Gleichbehandlung von qualifizierenden Studienabschlüssen ausgehebelt wurde. Soziale Gerechtigkeit wurde außer Kraft gesetzt, und Diplompsychologen mit 600 und mehr Stunden Ausbildung im klinischen Bereich während des Studiums, zusätzlichen klinischen Praktika etc. werden gravierend schlechter oder gar nicht entlohnt, während ärztliche Kolleginnen und Kollegen ohne spezifische Vorerfahrung bezüglich psychischer Störungen Gehälter bekommen, die über den üblichen Tariflöhnen von Personen mit universitären Abschlüssen liegen.

Auch wenn es in vielen Fällen zu positiven Erfahrungen im Kontext des Psychiatriejahres kommt, hat die aktuelle Regelung im Psychotherapeutengesetz zu einem Missbrauch von billigen Arbeitskräften mit schlechter sozialer Absicherung geführt. Eine Monopolstellung im Sinne eines zwingenden Vorschreibens von Psychiatriezeiten ist maximal für eine Zeitspanne von 3 Monaten gerechtfertigt, um entsprechende Krankheitsbilder kennenzulernen. Für die weitere Praxiszeit mag eine Tätigkeit in der Psychiatrie sinnvoll sein, unter Umständen wird jedoch in anderen Krankenhäusern mehr und bessere Psychotherapie gelernt, und es werden Erfahrungen mit Störungsbildern gesammelt, die für die spätere psychotherapeutische Praxis von mindestens gleicher Relevanz sind wie die Patientengruppen der Psychiatrie. Das Psychotherapeutengesetz muss sich stärker orientieren an dem Hauptziel der Psychotherapieausbildung: moderne, kompetente Psychotherapie zu erlernen bei einer Vielzahl von Störungsbildern und verschiedenen Schwierigkeitsgraden. Diese Zielsetzung sollte festgelegt werden (z.B. in Form von Diagnose-Katalogen, die gefordert sind), jedoch nicht über die ungerechtfertigte Bevorzugung eines spezifischen stationären Settings. Darüber hinaus sind sozial gerechte Lösungen erforderlich, die die akademische Vorqualifikation berücksichtigen.

Winfried Rief, Marburg

\section{Literatur}

Schulz H, Barghaan D, Harfst T, Koch U: Gesundheitsberichterstattung des Bundes, Heft 41. Psychotherapeutische Versorgung. Berlin, Robert Koch-Institut, 2008. 
mente: die dort gemachten Erfahrungen ließen sich auch andernorts sammeln, die Dauer von $1 \mathrm{Jahr}$ sei zu lang und die Psychologischen Psychotherapeuten in Ausbildung (PPiA)

\section{Das Psychiatriejahr ist unverzichtbar}

Im Psychotherapeutengesetz (PsychThG) ist festgelegt, dass als wichtiger Baustein in der Ausbildung zum Psychologischen Psychotherapeuten eine praktische Tätigkeit an psychiatrischpsychotherapeutischen Kliniken in einem Umfang von $1200 \mathrm{~h}$ über mindestens 12 Monate auszuüben ist. Auch wenn das sogenannte Psychiatriejahr damit der «größte Einzelposten im Katalog der geforderten Leistungen für die Psychotherapieausbildung» (Zitat Rief) ist, kann daraus kein pauschaler Vorwurf einer missbräuchlich ausgenutzten «Monopolstellung» der Psychiatrie abgeleitet werden. Denn das Psychiatriejahr ist zwar ein wichtiges, aber eben nur eines von mehreren Elementen im Rahmen der Psychotherapieausbildung für Psychologen, in dem man Inhalte lernt, die nur im Fach Psychiatrie und Psychotherapie zu erwerben sind.

Laut § 2 (1) der Ausbildungs- und Prüfungsverordnung für Psychologische Psychotherapeuten (PsychTh-APrV) besteht das mit dem Psychiatriejahr verbundene wichtige Ziel im «Erwerb praktischer Erfahrungen in der Behandlung von Störungen mit Krankheitswert ... sowie von Kenntnissen anderer Störungen, bei denen Psychotherapie nicht indiziert ist». Diese Formulierung ist natürlich absurd, anachronistisch und ignoriert die Integration von Psychotherapie, die in den letzten 20 Jahren in der Psychiatrie stattgefunden hat. Psychotherapie spielt als Behandlungsmethode im Rahmen eines mehrdimensionalen Behandlungskonzepts bei der Therapie aller psychischen Störungen eine zentrale Rolle, sodass eigentlich keine psychische Erkrankung vorstellbar ist, bei der Psychotherapie nicht indiziert wäre - und sei es als Ergänzung zu Pharmakotherapie. Hier muss der $\S 2$ (1) dringend dem heutigen Stand entsprechend umformuliert werden. Abgesehen davon, dass die bisherige Formulierung anachronistisch und absurd erscheint, ist es für die spätere Tätigkeit Psychologischer Psychotherapeuten jedoch äußerst wichtig, differentialdiagnostische Fähigkeiten zu erwerben und Entscheidungen über die Differentialindikation zu Psychotherapie als Einzel- oder Kombinationsverfahren zu treffen. Nur durch die Tätigkeit in einer psychiatrisch-psychotherapeutischen Klinik kann der angehende Psychologische Psychotherapeut in ausreichender Weise Kenntnisse über nicht psychotherapeutische Behandlungsverfahren erwerben und lernen, wann - vor allem bei Patienten mit schweren oder chronischen Erkrankungen - die Einbeziehung eines Facharztes für Psychiatrie und Psychotherapie oder die Einleitung einer stationären Behandlung notwendig werden bzw. wann Psychotherapie mit anderen Behandlungsmöglichkeiten wie Pharmakotherapie kombiniert werden muss.

Kritiker, die eine grundlegende Änderung oder gar Abschaffung des Psychiatriejahres in seiner bisherigen Form fordern, beziehen sich dabei im Wesentlichen auf drei Arguwürden von den betreffenden Einrichtungen als billige Arbeitskräfte ausgenutzt.

Sicher mag es in Einzelfällen auch andere Behandlungssettings geben, die das Kennenlernen eines ebenso breiten Diagnosespektrums erlauben würden. Dennoch steht außer Frage, dass nur die Tätigkeit in einer psychiatrisch-psychotherapeutischen Einrichtung, wie sie vom Gesetzgeber für das Psychiatriejahr vorgesehen ist, eine ausreichende Gewähr dafür bietet, dass tatsächlich sowohl psychische Erkrankungen in ihrer gesamten Vielfalt als auch alle besonders schwer ausgeprägten psychischen Erkrankungen und deren mitunter langwierigen Verlaufsformen von den in Psychotherapieausbildung befindlichen psychologischen Kollegen gesehen werden. Denn nur in diesen Einrichtungen werden diese Patientengruppen in ausreichender Zahl behandelt, während dies z.B. in einem ambulanten oder tagesklinischen Setting - auch bei gutem Willen aller Beteiligten - nicht realisierbar sein dürfte.

Die Forderung nach einer drastischen Verkürzung der praktischen Tätigkeit lässt außer Acht, dass stationäre psychiatrische Behandlungen durchschnittlich 6-8 Wochen, bei einigen Patientengruppen aber auch mehr als 10 Wochen erfordern. Sollen aber konkrete praktische Erfahrungen mit einem möglichst breiten Diagnosenspektrum gemacht werden, kann dies bei einer Verkürzung z.B. auf 3 Monate nicht mehr sichergestellt werden.

In einer psychiatrisch-psychotherapeutischen Klinik kann weiterhin beispielhaft das Zusammenwirken unterschiedlicher Berufsgruppen bei der Behandlung von psychisch erkrankten Patienten nach einem mehrdimensionalen Konzept (Psychotherapie, Pharmakotherapie, Soziotherapie) erfahren werden. Dies kann wesentlich zum besseren gegenseitigen Verständnis der einzelnen Berufsgruppen beitragen - und zwar sowohl in Bezug auf die gleichzeitige Behandlung ein und desselben Patienten, als auch in Bezug auf die im späteren Berufsalltag mit seinen unterschiedlichen Behandlungssettings (stationär, ambulant, Akutversorgung, Rehabilitation) auftretenden Schnittstellen. Nur an einem solchen Ort kann die Integration verschiedener therapeutischer Methoden erlernt und trainiert werden.

Das Psychiatriejahr muss selbstverständlich hohen qualitativen Ansprüchen genügen. In seiner bisher bestehenden Form bedarf es einer Reform, und der «Missbrauch», d.h. die Ausnutzung von PPiA als billige Arbeitskräfte muss ausdrücklich ausgeschlossen werden. Dies betrifft neben den psychiatrischen Kliniken im Übrigen mindestens genauso psychosomatische Kliniken, die PPiA in großem Umfang als billige Arbeitskräfte anstellen. So müssen PPiA während ihrer Tätigkeit an psychiatrischen und psychosomatischen Kliniken angemessen bezahlt werden. PPiA werden in psychiatrischen Kliniken als kompetente Mitarbeiter angesehen, welche die praktische, 
aber auch die wissenschaftliche Arbeit, zum Beispiel in Universitätskliniken, mit ihrer akademischen und zum Teil auch praktischen Vorerfahrung wesentlich bereichern. Diese Tätigkeit muss einheitlich und angemessen vergütet werden, was in ca. der Hälfte der Kliniken auch bereits geschieht. Auch wenn persönliche Erfahrungen nur einen kleinen Ausschnitt der Realität widerspiegeln - dies gilt sowohl für Herrn Riefs als auch für meine persönlichen Erfahrungen - so muss ich doch feststellen, dass die weitaus meisten PPiA, die an der Lübecker Klinik (natürlich gegen Bezahlung) tätig sind, das Psychiatriejahr als ausgesprochen sinnvollen und prägenden Teil ihrer Ausbildung erleben und nicht missen wollen.

Neben der praktischen Tätigkeit steht die im günstigen Falle damit eng verzahnte und aufeinander abgestimmte strukturierte und curriculare Ausbildung in Psychotherapie, die ohnehin strengen Qualitätsstandards unterliegt.

Da die Ausgestaltung des Psychiatriejahres in der Verantwortung der jeweiligen Ärzte liegt, die über die psychiatrisch-psychotherapeutische Weiterbildungsbefugnis verfügen, unterscheidet sich die praktische Durchführung des Psychiatriejahres von Klinik zu Klinik jedoch mitunter sehr. Die Deutsche Gesellschaft für Psychiatrie, Psychotherapie und Nervenheilkunde (DGPPN) hat deshalb Empfehlungen an Chefärzte psychiatrisch-psychotherapeutischer Kliniken zur Gestaltung und Organisation der praktischen Tätigkeit während der Ausbildung zum Psychologischen Psychotherapeuten formuliert [Gaebel, 2008; DGPPN, 2009]. Dabei wird deutlich, dass das Psychiatriejahr, sowohl was den geeigneten Einrichtungstyp als auch dessen Dauer betrifft, nicht verändert werden sollte. Bezüglich der Vergütung der PPiA und der konkreten inhaltlich-organisatorischen Ausgestaltung der Tätigkeit gibt es jedoch durchaus Reform- und Optimierungsbedarf. Die Frage ist also nicht - Psychiatriejahr: Ja oder Nein -, sondern vielmehr, wie das Psychiatriejahr gestaltet werden muss, um eine optimale Ausbildung und damit spätere Patientenbehandlung zu gewährleisten.

Fritz Hohagen, Lübeck

\section{Literatur}

DGPPN (Hrsg): Stellungnahme der Deutschen Gesellschaft für Psychiatrie, Psychotherapie und Nervenheilkunde (DGPPN) zum «Forschungsgutachten zur Ausbildung zum psychologischen Psychotherapeuten und zum Kinder- und Jugendlichenpsychotherapeuten». Berlin, DGPPN, 2009.

Gaebel W: Empfehlungen der DGPPN an Chefärzte psychiatrisch-psychotherapeutischer Kliniken zur Gestaltung der praktischen Tätigkeit während der Ausbildung zum Psychologischen Psychotherapeuten. Nervenarzt 2008;5:632-634. 